\section{P2.117 EFFECT OF HAART ON GASTRROINTESTINAL AND HEPATOBILIARY OPPORTUNISTIC INFECTIONS}

doi:10.1136/sextrans-2013-051184.0381

J J Toshniwal, M Sachdeva, T Singh. Sir Gangaram Hospital, New Delhi, India

Background Opportunistic infections (OI) of gastrointestinal (GI) and hepatobilliary system (HB) are common in HIV infected patients. Despite highly active antiretroviral therapy (HAART) GI OI have been reported in HIV infected patients. There is paucity of data from south Asia describing occurrence of GI and $\mathrm{HB} O \mathrm{OI}$ in AIDS with use of HAART.

Method Study population included 74 HIV infected patients (Male $=57$, Female $=17$ ) in the age group of 17 to 63 years admitted to a tertiary care referral centre in North India from January 2011 through December 2012. Only subjects who presented with GI and $\mathrm{HB}$ system manifestations were enrolled in the study. 74 study subjects were stratified into HAART naïve (36) and HAART experienced (38) groups according to their HAART status on admission. HIV infection was confirmed by western blot test. Gastrointestinal and hepatobilliary pathologies including $\mathrm{OI}$ were diagnosed and defined as per standard protocols.

Results In HAART experienced group 33\% and in HAART naïve group $52 \%$ patients were diagnosed with OI. Esophageal candidiasis was present in $10 \%$ patients in HAART experienced group and in $7 \%$ patients in HAART naïve group ( $p$ value $>0.05$ ). Abdominal Tuberculosis was present in $24 \%$ patients in HAART experienced group and in $33 \%$ patients in HAART naïve group ( $p$ value $>0.05$ ). Drug induced liver injury was present in 10 patients in HAART experienced group and in 5 patients in HAART naïve group.

Conclusion In our study we conclude that Gastrointestinal and Hepatobiliary OI are equally common in patients taking HAART; especially esophageal candidiasis and abdominal tuberculosis, reasons for this include HAART resistance, drug noncompliance, immune restoration inflammatory syndrome (IRIS) and high prevalence of tuberculosis in this region.

\section{P2.118 THE IMPORTANCE OF HIV TESTING DURING PREGNANCY - A CASE REPORT}

doi:10.1136/sextrans-2013-051184.0382

K Jahn, P Brunner, C Schuster, A Rieger. Department of Dermatology, Div. of Immunology, Allergy and Infectious Diseases, Medical University of Vienna, Austria

A 7 year old Austrian girl presented with a 20-month history of oral thrush as well as onychomycosis of the hands. In addition, dysphagia suggested esophageal involvement. Her family history was negative for chronic mucocutaneous candidiasis (CMC). Prior treatments with systemic antimycotic agents (miconazole, amphotericin B) showed only little relief and were followed by immediate relapses. The initial differential diagnoses were CMC as well as other forms of chronic immunosuppression, including an underlying malignancy such as lymphoma, HIV/AIDS and tpye 1 diabetes mellitus. The initial laboratory examination revealed a positive HIV-ELISA and Western blot, a viral load of 31.000 copies/ml and a severe CD4 cell depletion $\left(94 / \mathrm{mm}^{3}\right)$, leading to the diagnosis of HIV in childhood. After getting an overview about the family situation we realised that the mother had been neglecting her known HIV infection and that the little girl had never been tested before.

Conclusion Screening for HIV infection during pregnancy is the key prerequisite for the prevention of vertical HIV transmission. Testing within the first 16 weeks of pregnancy is obligatory since 2010 according to the Austrian prenatal care guidelines. This case report emphasises the importance of HIV testing during pregnancy. The younger sister of our patient had already benefitted from these guidelines because the mother had been tested positive for HIV for the first time during her third pregnancy, and mother-to-child transmission was prevented by the timely initiation of antiretroviral treatment of the maternal HIV infection.

\section{P2.119 THE PREVALENCE OF HEARING SENSITIVITY AMONG HIV-SEROPOSITIVE AND HIV-SERONEGATIVE MEN AND WOMEN}

doi:10.1136/sextrans-2013-051184.0383

${ }^{1} \mathbf{M}$ W Plankey, ${ }^{2} \mathrm{H}$ J Hoffman, ${ }^{3} \mathrm{G}$ Springer, ${ }^{3} \mathrm{C}$ Cox, ${ }^{1} \mathrm{M}$ A Young, ${ }^{3} \mathrm{~J} B$ Margolick, ${ }^{4} \mathrm{P}$ Torre. 'Georgetown University Medical Center, Washington, DC, United States; ${ }^{2}$ National Institute of Deafness and Communication Disorders, Bethesda, MD, United States; 3Johns Hopkins University Bloomberg School of Public Health, Baltimore, MD, United States, ${ }^{4}$ San Diego State University, San Diego, CA, United States

Background We measured the prevalence of hearing sensitivity among HIV + and HIV- men and women and identified associated risks co-factors.

Methods Audiometric testing was conducted among 262 men [median age 54.7 years; 117 (44.7\% HIV+; median (25th, 75th): nadir CD4+: 296 (191, 400); viral load (VL): 40 (40.40))] from the Baltimore-DC site of the Multicenter AIDS Cohort Study and 134 women [median age 45.2 years; 105 (78.4\% HIV+; median (25th, 75th): nadir CD4+: 249 (92, 367); VL: 80 (48,1270))] from the DC site of the Women's Interagency HIV Study. Pure-tone hearing thresholds were obtained at 500,1000, 2000, and $4000 \mathrm{~Hz}$ and HL was defined as a pure tone average (PTA) $\geq 20 \mathrm{~dB}$ hearing level in either ear. A linear mixed model with a random-subjects effect was used to account for two repeated measurements (one per ear) adjusted for age, gender, race, HIV status, and noise exposure. The $\mathrm{HIV}+$ model included nadir CD4+, peak CD8+, VL, ever having AIDS, ever monotherapy (MT), ever combination therapy (CT) and ever HAART use.

Results 84 (95.2\% men, 4.8\% women) HIV- and 90 (65.6\% men, $34.4 \%$ women) HIV + participants had HL in the poorer ear. Age was a statistically significant risk factor of HL, however HIV status and noise exposure were not. In the HIV+ model, nadir CD4+, peak CD8+, VL, ever having AIDS, and MT were not statistically significantly associated with HL. Although there was a higher PTA and a lower PTA with ever CT and HAART use neither was significantly associated with $\mathrm{HL}$.

Conclusions We found no impact of HIV status or treatment variables on HL. HIV-infected individuals who used HAART had a lower PTA, an indicator of better hearing sensitivity. However, due to cross-sectional design of this study, it is not known whether HAART use protects hearing sensitivity.

\section{P2.120 INCIDENCE AND RISK FACTORS OF HERPES ZOSTER AMONG HIV-POSITIVE PATIENTS IN THE COHORT OF THE GERMAN COMPETENCE NETWORK FOR HIV/AIDS (KOMPNET)}

doi:10.1136/sextrans-2013-051184.0384

\begin{abstract}
${ }^{1,2} \mathrm{C}$ Michalik, ${ }^{1,3} \mathrm{~K}$ Jansen, ${ }^{4} \mathrm{~B}$ Haastert, ${ }^{1,5} \mathrm{~S}$ Dupke, ${ }^{1,6} \mathrm{~S}$ Esser, ${ }^{1,7} \mathrm{~A}$ Plettenberg, ${ }^{1.8} \mathrm{~S}$ Köppe, ${ }^{9} \mathrm{~A}$ Guignard, ${ }^{1,3} \mathbf{A}$ Skaletz-Rorowski, ${ }^{1,3,10} \mathrm{~N}$ H Brockmeyer. ${ }^{1}$ Competence Network for HIVIAIDS, Ruhr-Universität Bochum, Bochum, Germany; ${ }^{2}$ Centre for Clinical Trials, Cologne, Germany; ${ }^{3}$ Clinic for Dermatology, Venerology and Allergology, RuhrUniversität Bochum, Bochum, Germany; ${ }^{4}$ mediStatistica, Neuenrade, Germany; ${ }^{5}$ Private practise Driesener Strasse, Berlin, Germany; ${ }^{6}$ Clinic for Dermatology and Venerology, University Clinic, Essen, Germany; 'Ifi-Institute, Asklepios-Clinic, Hamburg, Germany; ${ }^{8}$ Medical Practice Mehringdamm, Berlin, Germany; ${ }^{9} G S K$ Biologicals, Wavre, Belgium; ${ }^{10}$ German STI-Society (DSTIG), Bochum, Germany
\end{abstract}

Background HIV infection is a risk factor for development of Herpes Zoster (HZ) and its complications. There is limited evidence on the impact of antiretroviral therapy (ART) on the occurrence of $\mathrm{HZ}$ among HIV-infected adults. 